sampled serially showed little change with time.

Nerve conduction studies showed normal motor latencies, conduction velocities and $F$ wave latencies. The ulnar sensory nerve action potentials and mixed afferent nerve potentials were of reduced amplitude but also had normal conduction times, suggesting an axonal degeneration.

A biopsy of the deltoid and quadriceps muscles showed non-specific changes only and no dystrophic features.

In February 1989 an MRI scan of the cervical spine showed the upper cervical cord to have a slightly increased signal intensity on the T2 weighted spin echo sequence, the significance of which was unclear.

This patient developed an asymmetrical and patchy wasting and weakness of the shoulder girdle muscles involving several myotomes from $\mathrm{C} 3$ to $\mathrm{C} 7$. The explanation for the symptomless minimal weakness of the orbicularis oculi and frontalis muscles is uncertain. It may reflect patchy involvement of the brainstem (similar symptomless involvement of the other cranial nerves such as the bulbar muscles would escape detection) or might merely be constitutional.

We postulate that the neurological abnormalities in our patient are a manifestation of augmentation of irradiation by combination intrathecal and systemic chemotherapy with two potentially neurotoxic agents, methotrexate and cytosine arabinoside, in accordance with the intensive UKALL 4 regimen. The predominant features of this case are in keeping with a postradiation motor neuron syndrome.' The minor ulnar sensory abnormalities detectable only on EMG and without associated clinical symptoms or signs were probably secondary to vincristine.

Four types of radiation myelopathy have been described, ${ }^{2}$ the least common being lower motor neuron syndrome. The mechanism underlying this phenomenon is unclear but there is evidence from clinical and pathological studies that radiation injury to vascular endothelium produces ischaemia which leads to selective anterior horn cell degeneration. ${ }^{3-5}$ Greenfield and Stark observed this phenomenon in three patients, and Sadowsky et al reported it in a fourth. ${ }^{16}$ All four cases consisted of a selective lower motor neuron disease confined to lower limb muscles starting three to eight months after radiation of the spinal axis. All followed a subacute and selflimiting course. In our patient the latent period was longer ( $21 / 2$ years), in keeping with other studies where the average symptom free interval was 14 months, ${ }^{2}$ but otherwise the disease followed a similar course. Unlike the other cases described, he did not receive direct irradiation to the spinal cord apart from that part of the upper cervical cord included in the field during cranial irradiation.

Byfield described an infant who developed radiation myelitis of the cervical cord after receiving routine doses of vincristine and radiation therapy and postulated a synergistic effect. ${ }^{7}$ In 1975 a histopathological study by Price et al suggested a similar synergism between irradiation and intravenous methotrexate in the development of fatal leukoencephalopathy in children with ALL. ${ }^{8}$ Intrathecal methotrexate has a direct neurotoxic effect ranging from a chemical arachnoiditis to transient/permanent paresis and encephalopathy.

Cytosine arabinoside, the other intrathecal agent used in our patient, can cause disseminated multifocal coagulation necrosis of white matter and has been implicated in enhancing radiation induced chromosomal abnormalities.' A recent study has shown that intrathecal ara-C significantly reduces the isoeffect doses required for the development of radiation damage in rat spinal cord. ${ }^{10}$

We are not aware of any previous reports describing an upper cervical cord motor neuron syndrome occurring following cranial irradiation and would be interested to hear if others have encountered this feature in leukaemic patients treated similarly.

SV TAN Leicester Royal Infirmary,
Leicester, UK

Figure CT scan shows thickened retrobulbar

Correspondence to: $\mathrm{Dr}$ Tan, 21 Avon Road Leicester LE3 $3 \mathrm{AB}$, UK

1 Sadowsky CH, Sachs E, Ochoa J. Postradiation motor neurone syndrome. Arch Neurol 1976; 33:786-7.

2 Reagan TJ, Thomas JE, Colby MY. Chronic progressive radiation myelopathy. JAM 1968;203(2): 128-32.

3 Jellinger K, Sturm KW. Delayed radiation myelopathy in man. $J$ Neurol Sci 1971;14: 389-408.

4 Palmer JJ. Radiation myelopathy. Brain 1972; 95:109-22.

5 Dodson WE, Landau WM. Motor neurone loss due to aortic clamping in repair of coarctation. Neurology 1973;23:539-42.

6 Greenfield MM, Stark FM. Post-irradiation neuropathy. Am $J$ Roentgenol Radium Ther Nucl Med 1948;60:617-22.

7 Byfield JE. Ionising radiation and vincristine: possible neurotoxic synergism. Radiol Clin Biol 1972;41:129-38.

8 Price JA, Jamieson PA. The Nervous System in childhood leukaemia II. Subacute leukoencephalopathy. Cancer 1975;35:306.

9 Ejima Y, et al. Enhanced expression of X-ray and $U V$-induced chromosome aberrations by cytosine arabinoside in ataxia telangiectasia cells. Mutat Res 1986;159(1-2):117-23.

10 Van der Kogel AJ, Sissingh HA. Effects of intrathecal methotrexate and cytosine arabinoside on radiation tolerance of the rat spinal cord. Radiother Oncol 1985;4(3): 239-51.

\section{Optic nerve cysticercosis: a case report}

Cysticercosis is caused by infection from the larval form of Taenia solium and humans are an intermediate host. The subcutaneous tissue, brain, eye, muscle, heart, liver and peritoneum are common sites of encystment. In the eye, the conjuctiva is the most common site. Subretinal and intravitreal locations have also been described. ${ }^{2}$ Cysticercosis of the intracranial portion of the optic nerve is rare.

A 15 year old school girl presented with deteriorating vision in her right eye which had progressed over a seven month period. There was no history of pain or inflammation in the eye, trauma, headache, vomiting, seizures, or tuberculosis. General physical and systemic examinations were normal There was no subcutaneous nodule or cafeau-lait spots. Neurological examination was also normal except for the patient's inability to count fingers at less than $30 \mathrm{~cm}$ with the right eye. The pupils were equal and reacting. Fundus oculi examination revealed disc pallor in the upper half, disc margins elevated in the lower part and neighbouring retina for an area of two dioptres below the disc. Vessels and macula were normal. Pigmentary changes were present. The tension was $17.3 \mathrm{~mm}$ in both eyes. Other cranial nerves were normal. The rest of the neurological examination did not reveal any abnormality. segment of right optic nerve with small area of low attenuation in the thickened portion.

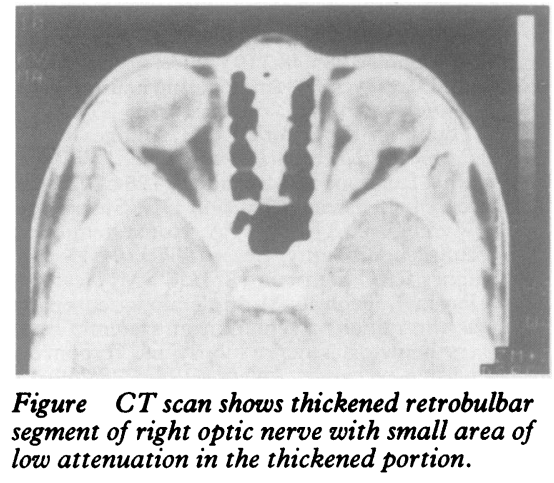
meters were normal. The erythrocyte sedimentation (ESR) was $26 \mathrm{~mm}$ and the VDRL was negative. Skull radiographs of the optic foramen and superior orbital fissures were normal. CT scan of the head and orbit with contrast enhancement showed a retrobulbar segment of the right optic nerve thickened with a small area of low attenuation in the thickened portion of the optic nerve Retrobulbar fat was preserved and the muscle cone was normal. The optic nerve at the orbital apex appeared to be of normal width. The brain parenchyma was normal as was the left optic nerve (fig). Perimetry revealed superior altitudinous right hemianopia Ultrasonography showed a mass in the region of the right optic nerve. The Casoni test was negative.

A diagnosis of optic nerve glioma or granuloma was considered. A right frontal craniotomy and extradural frontal orbitomy was performed. After incising the tenon's fascia normal retrobulbar fat protruded. The optic nerve was exposed by microdissection and was found to have fusiform thickening. A small portion of nerve just behind the optic globe was normal as was nerve near the apex. Longitudinal incision was made over the maximum bulge. There was intense fibrosis On deeper incision a sago grain like cyst was found and excised. Histopathology revealed it to be cysticercosis. Postoperatively the patient's vision fully recovered but there was IIIrd nerve paresis.

Cysticercosis is one of the most serious public health problems in the developing countries. ${ }^{3}$ Any part of the neuraxis can be involved, except the peripheral nerves, resulting in protean features. ${ }^{4}$ Ocular cysticercosis occurs in 3\% of cases and may be single, unilateral or bilateral. ${ }^{5}$ Subretinal involvement of the eye usually occurs initially through the posterior ciliary arteries but migration of the parasite is common. The nasal side of the eye is more commonly involved than the lateral side. This is due to an anatomical peculiarity of the ophthalmic artery which after giving rise the lacrimal branch runs along the medial side of the orbit and divides into its terminal branches. ${ }^{2}$ The optic nerve obtains its blood supply from the branch of the central artery of the retina and retinal blood vessels may thus be involved. of the eyes due to iridocyclitis and dimness of vision. The eye may be involved alone or may be associated with other clinical features of neuro-cysticerosis when the brain is also involved.

Optic nerve involvement is rare in any kind of cyst or granuloma. As a result, in our
The usual symptoms are of pain, irritation 
patient the lesion in the retro orbital portion of the right optic nerve was thought to be an inflammatory granuloma or glioma cosis. To our knowledge this has not been described previously.

V S MADAN H S GILL M S BOPARAI P D SOUZA P C SANCHETEE J R BHARDWAJ The Army Hospital, Delhi, and the Lady Harding Medical College, New Delhi, India Correspondence to: Dr Madan, Department of
Neurology, Sir Ganga Ram Hospital, Rajinder Neurology, Sir Ganga Ram Helhi-110060, India

1 Baranski MC. Teniasis. In: Chaia G, Ed. Atlas of parasitology. Sao Paulo: Johnson and Johnson Research Institute for Endemic Diseases, 1975:69-76.

2 Malik SRK, Gupta AK, Choudary S. Ocular cysticercosis. Am J Ophthalmol 1968;66: 1166-71.

3 Mingnetti G, Ferreira MVC. Computed tomography in neurocysticercosis. $J$ Neurol Neurosurg Psychiatry 1983;46:936-42. cosis. In: Ahuja MMS, ed. Progress in clinical medicine in India, Series Four New Delhi: medicine in India, Serinemann, 1981:382-406.

5 Kapoor S, Sood GC, Aurora AL, Sood M. Ocular cysticosis, report of a free floating Ocular cysticosis, report of a free floating
cysticercus in the anterior chamber. Acta Ophthalmologica 1977;55:927-30.

\section{Vertebral haemangioma causing acute} recurrent spinal cord compression

Vascular tumours are commonly seen in bone, particularly in vertebral bodies; they are, however, far less common in the epidural space. Most of these epidural tumours represent extension of vertebral lesions. Patients with epidural lesions usually present with slowly progressive symptoms of back pain or compression of the spinal cord. Our patient presented with neurological symptoms that developed with unusual rapidity and seemed to be recurrent. This clinical picture has not to our knowledge previously been described. preoperatively but it proved to be cysticerR M DHAMIJA

4 Venkataraman S, Vijayan GP. Neurocysticer-

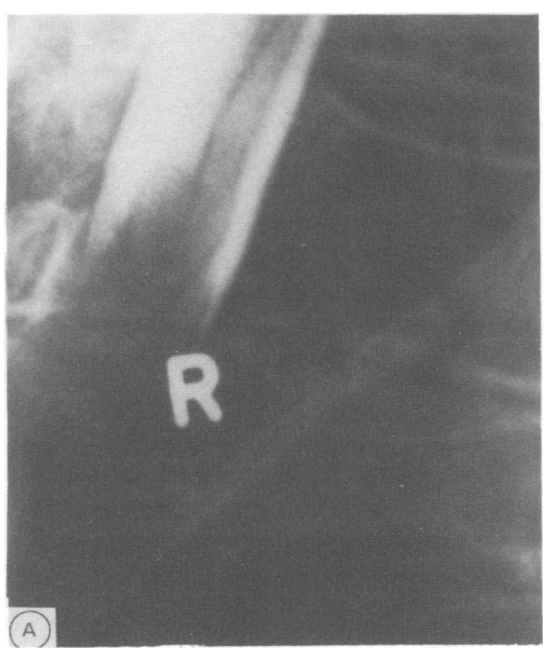

A 73 year old woman had difficulty in walking. Ten years earlier, the patient had had a gradual onset of stiffness in both legs. Results of neurological examination and radiography of the spine and chest were normal. A non-organic mechanism was considered. The symptoms improved over three months, and the patient remained asymptomatic for nine years.

Six months before the present examination, the patient experienced gradual unsteadiness of gait. Five days before this evaluation, she experienced mild difficulty lifting her right foot. On admission, she was unable to walk without assistance. Examination showed mild paraparesis. Exaggerated patellar reflexes and Babinski's signs were bilaterally present. Sensory examination disclosed a mild deficit to soft touch on the right leg below the knee.

Results of routine blood studies and CT of the head were normal. Radiography of the spine demonstrated severe compression at T6 and diffuse osteoporosis. Review of the findings on chest radiography obtained 10 years earlier revealed a compression at T6 that had not been observed originally; however, this status was unchanged (fig 1A)

On the day after admission, bilateral sensory impairment developed below the T6-7 level. Myelography demonstrated complete block at T6 (fig 1A). Total laminectomy at T4-6 demonstrated a highly vascular epidural tumour extending from the affected T6 vertebra, which was excised. Pathological evaluation revealed a cavernous haemangioma (fig 1B).

Sensation and strength returned to both legs, and six days after the operation, the patient walked with assistance. She recovered well at a rehabilitation hospital.

Vertebral haemangioma that does not involve the epidural space is a common, usually asymptomatic, lesion. In Töpfer's ${ }^{1}$ series of 2154 patients, the incidence of vertebral haemangioma was $11.9 \%$. In a series of more than 10000 necropsies by Junghanns, ${ }^{2}$ the incidence was $10 \%$. Extension into the epidural space is uncommon. In a comprehensive study, Hurth ${ }^{3}$ estimated that $12 \%$ of all intraspinal haemangiomas involve the epidural space and suggested that these lesions are underdiagnosed. Most symptomatic epidural and vertebral haemangiomas involve the thoracic spine, ${ }^{4}$ possibly because the spinal canal is narrow at this level.

Rapid progression of symptoms associated with an epidural vascular lesion is a rare occurrence. Four pathophysiological mechanisms have been postulated ${ }^{56}$ that may lead to spinal cord compression: extension into the epidural space; expansion of the vertebrae, which causes narrowing of the spinal canal; epidural haemorrhage, possibly involving thrombosis; and compression fracture. We suggest that the most recent presentation in our patient fits the first or second category best because no new fracture was present and no acute bleeding or obvious thrombosis was found at operation.

We did not have the benefit of nuclear MRI at the time this patient was treated. Today, this procedure might well be the optimal method of imaging a lesion before operation. Some authors ${ }^{5}$ suggest that angiography be performed in less acute situations to permit embolisation of the lesion preoperatively.

Reports of neurological symptoms that developed as quickly as they did in our patient form a small minority of cases. We have not found a case previously described in which symptoms of subacute spinal cord compression developed and then resolved completely for 10 years, only to recur in an acute fashion as a result of an epidural haemangioma. Unsuspected epidural or vertebro-epidural vascular tumour, which may be recurrent, is a rare but treatable cause of acute spinal cord compression.

ONATHAN NEWMARK * H ROYDEN JONES Jr Department of Neurology CHRISTINE B THOMAS H THOMAS ARETZ Department of Anatomic Pathology STEPHEN R FREIBERG Department of Neurosurgery
RICHARD A BAKER Department of Diagnostic Radiology, Lahey Clinic Medical Center, Burlington, MA,USA $\star$ Present address: Hospital of the University of Pennsylvania, Neuromuscular Division, 3 Gates Building West,

Correspondence to: Dr Jones Jr, Department of Correspondence to: Dr Jones Jr, Department of Rourology, Lurlington, MA 01805, USA.

1 Töpfer D. Infiltrating hemangioma of skin and multiple capillary ectasias: angioma of vertebra. Frankfurt Ztschr $f$ Path 1928;36: $337-45$.

2 Junghanns $\mathbf{H}$. Hämangiom des 3 . Brustwirbelkörpers mit Rückenmark-kompression. Laminektomie. Heilung. Arch $f$ klin Chir 1932;169:321-30.

3 Hurth M. Les hemangioblastomes intrarachidiens. Neurochirurgie 1975;21(suppl 1): 1-136. (Eng abstr)

4 McAllister VL, Kendall BE, Bull JW. Symptomatic vertebral haemangiomas. Brain 1975;98:71-80.

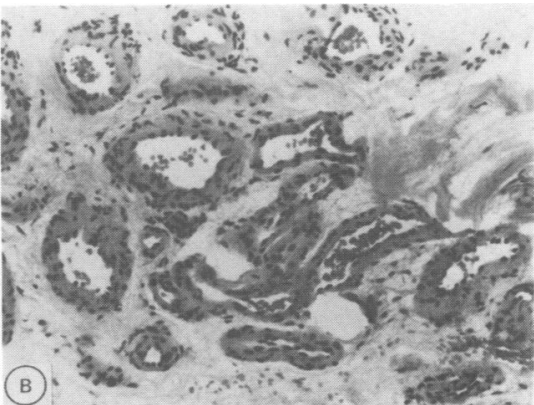

5 Graham JJ, Yang WC. Vertebral hemangioma with compression fracture and paraparesis treated with preoperative embolization and vertebral resection. Spine 1984;9:97-101.

6 Padovani R, Tognetti F, Proietti D, et al. Extrathecal cavernous hemangioma. Surg Neurol 1982;18:463-5.

Figure $1 A$ Lateral myelograph from above demonstrates total block at the level of the compressed T6 vertebra consistent with an epidural process; (B) section from the epidural component of the tumour shows large thick-walled vessels. Bone involvement was seen in other sections. (HEE $E$; original magnification $\times 100$.
Giant intracranial aneurysm associated with Marfan's syndrome: a case report

We report a case of Marfan's syndrome presenting with the thromboembolic or mass effects of a giant basilar aneurysm: this was successfully clipped by direct exposure, an outcome we believe not previously described in Marfan's syndrome. 